\title{
Stability of black hole accretion disks
}

\author{
A. Janiuk ${ }^{1, a}$, R. Misra ${ }^{2}$, B. Czerny ${ }^{3}$, and M. Kunert-Bajraszewska ${ }^{4}$ \\ ${ }^{1}$ Center for Theoretical Physics, Polish Academy of Sciences, Warsaw, Poland \\ ${ }^{2}$ Inter-University Center for Astronomy and Astrophysics, Pune, India \\ ${ }^{3}$ Nicolaus Copernicus Astronomical Center, Warsaw, Poland \\ ${ }^{4}$ Nicolaus Copernicus University, Torun, Poland
}

\begin{abstract}
We discuss the issues of stability of accretion disks that may undergo the limit-cycle oscillations due to the two main types of thermal-viscous instabilities. These are induced either by the domination of radiation pressure in the innermost regions close to the central black hole, or by the partial ionization of hydrogen in the zone of appropriate temperatures. These physical processes may lead to the intermittent activity in AGN on timescales between hundreds and millions of years. We list a number of observational facts that support the idea of the cyclic activity in high accretion rate sources. We conclude however that the observed features of quasars may provide only indirect signatures of the underlying instabilities. Also, the support from the sources with stellar mass black holes, whose variability timescales are observationally feasible, is limited to a few cases of the microquasars. Therefore we consider a number of plausible mechanisms of stabilization of the limit cycle oscillations in high accretion rate accretion disks. The newly found is the stabilizing effect of the stochastic viscosity fluctuations.
\end{abstract}

\section{INTRODUCTION}

The evolution and significant morphological changes in active galactic nuclei and their host galaxies may result from catastrophic events, such as mergers with other galaxies or tidal disruptions. Another type of processes that trigger the evolution of AGN are connected with intrinsic properties of their nuclei and do not have to involve the companion interaction or any violent catastrophes. In particular, the evolution of black hole accretion disk, believed to be a power house of quasars and AGN, may lead to secular changes in the observed properties of the source.

In this proceeding, we discuss the physics of processes in the accretion disk around compact star that under certain conditions lead to the thermal-viscous instability and periodic outbursts of luminosity in the AGN central engine. We also present observational support for the theoretical models of the disk instability as well as we discuss some possible mechanisms that may act on stabilizing the system. Because the physics is the same for a broad range of masses of the accreting black holes, we include in this discussion the results obtained for stellar mass black holes in binary systems. They are much easier to observe during the lifetime of our currently available instruments.

\section{AGN OUTBURSTS INDUCED BY ACCRETION INSTABILITIES}

The outbursts of accretion disks may be induced by the two main types of instabilities which lead to the thermalviscous oscillations. These are the radiation pressure instability and the partial hydrogen ionization instability, both known for over 40 years in theoretical astrophysics.

\footnotetext{
a e-mail: agnes@cft.edu.pl
}

For a detailed discussion of these instabilities the reader is referred to the recent article [5] as well as to the literature quoted therein. Below, we only briefly describe the fundamental physics behind these processes.

\subsection{Radiation pressure instability: Basic physics}

The stationary, thin accretion disk model in classical theory is based on $\alpha$ prescription for the viscous energy dissipation. In the $\alpha$-model we assume the non-zero component $T_{r \phi}$ of the stress tensor is proportional to the total pressure. The latter includes the radiation pressure component which scales with temperature as $T^{4}$ and blows up in hot disks for large accretion rates. This in turn affects the heating and cooling balance, between the energy dissipation and radiative losses.

Such a balance, under the assumption of hydrostatic equilibrium, is calculated numerically with a closing equation for the locally dissipated flux of energy given by the black hole mass and global accretion rate. The local solutions may be conveniently plotted on the so-called stability curve, shown in Figure 1. The scheme consists of distinct points which represent the annulus in an accretion disk with temperature and surface density determined by the accretion rate. If the accretion rate is small, i.e. induced temperature is between the points $\mathrm{A}$ and $\mathrm{C}$ on this scheme, the local annulus of the disk is gas pressure dominated and stable. For larger accretion rates, and temperatures between points $\mathrm{A}$ and $\mathrm{B}$, the annulus will be dominated by radiation pressure and unstable. The larger the global accretion rate, the more annuli of the disk will be affected by the instability and the extension of the instability zone grows in radius, starting around the inner edge of the disk which is the hottest.

If there was no stabilizing mechanism, the radiation pressure dominated disk would not survive. This is because in such parts of the disk the decreasing density leads to the 


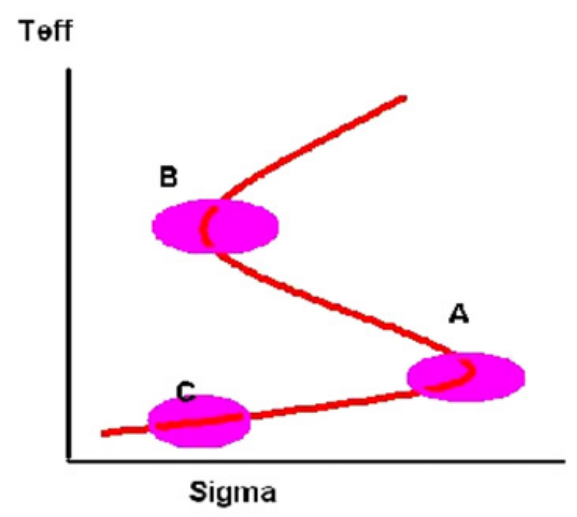

Figure 1. Schematic solutions of the local thermal balance in the accretion disk, shown on the surface density-temperature plane.

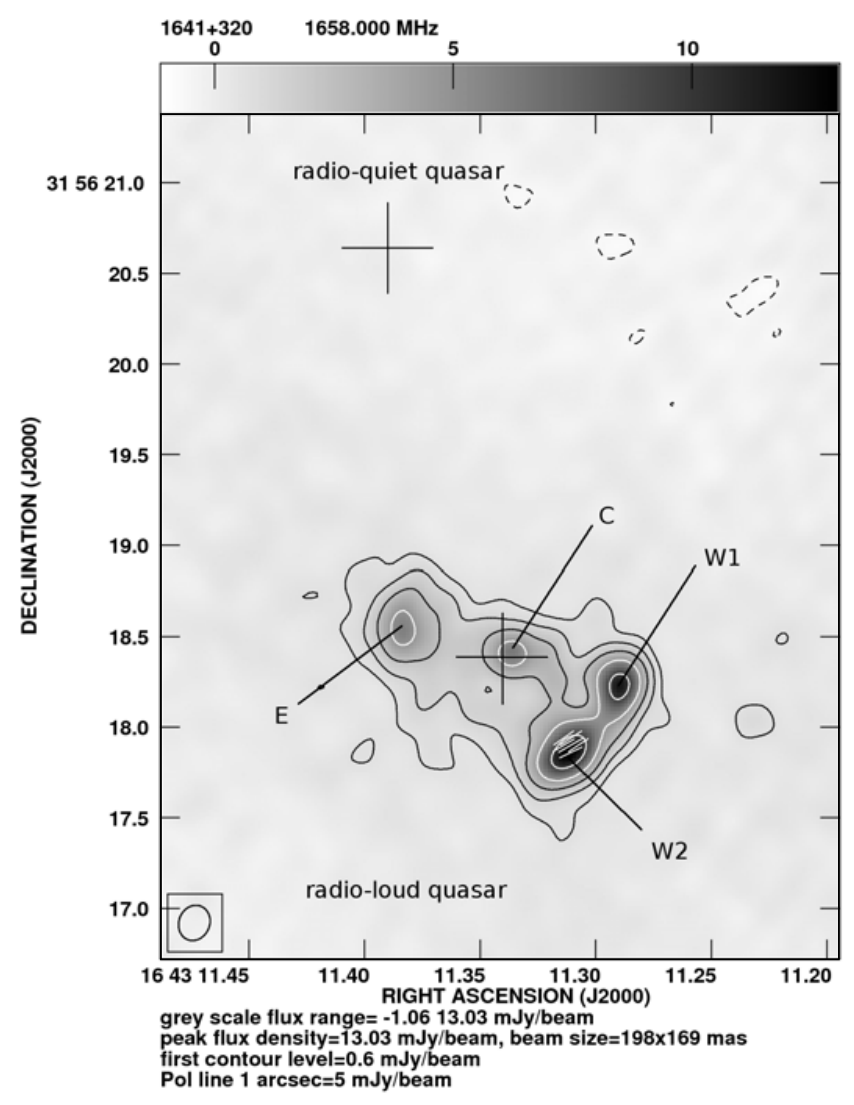

Figure 2. MERLIN radio image at $1.66 \mathrm{GHz}$ of the binary quasar FIRST $1641+320$ [7]. The pair consists of the radio-loud and radio-quiet sources. Their positions found in the SDSS are marked by crosses. Contours increase by a factor of two and the first contour level corresponds to $\sim 3 \sigma$. The symbols indicate: $\mathrm{C}$ - radio core, $\mathrm{E}$ - eastern jet, $\mathrm{W} 1, \mathrm{~W} 2$ - western jet.

temperature growth. In consequence, the local accretion rate increases and more material is transported inwards. The disk annulus empties because of both increasing accretion rate and decreasing density, so there is no selfregulation of the disk structure. However, the so called 'slim-disk' solution, where advection of energy provides additional source of cooling in the highest accretion rate regime (close to the Eddington limit), acts as a stabilizing branch. Therefore, even if a large part of the disk is dominated by radiation pressure, advection of some part of energy allows the disk to survive and oscillate between the hot and cold states. Such oscillating behavior leads to periodic changes of the disk luminosity, as shown e.g. in [4] for the black hole X-ray binary disk. The advective solutions constitute the upper stable sequence on the $S$-curve shown in Figure 1.

\subsection{Partial hydrogen ionization instability}

The ionization instability operates in the outer regions of the accretion disk, where the temperatures are in the range of $\log T=3.5-4[\mathrm{~K}]$. This is the temperature range where Hydrogen is partially ionized. As known from the stellar astrophysics and dwarf novae theory, under such conditions the opacities in the plasma depend inversely on density and temperature, which in turn affects the local thermal balance, somewhat similarly to the radiation pressure discussed above.

The crucial difference between stellar black hole disks and AGN disks is that the latter are cooler, as their global properties scale with the black hole mass. The AGN disks, which radiate mostly in the UV range, have therefore the hydrogen ionization zone much closer to the black hole than the BHXB disks (for the distance expressed in Schwarz-schild radii). In the latter, the ionization instability zone may never form, if the disk size, determined by the binary system separation and orbital period, is too small. It may happen that the whole disk is too hot for that [5].

\subsection{Overlapping of the instability zones}

The limitation by the disk size does not apply to the radiation pressure instability. In consequence, at high accretion rates, the AGN disks may have two instability zones close to each other, while in GBH there may be only the radiation pressure dominated unstable zone. On the other hand, for small accretion rates, the AGN disks will still have the ionization instability zone, while the GBH disks may be stable to whatever type of instability if they are small in radius.

\section{OBSERVATIONAL FACTS}

The studies of AGN evolution are difficult with respect to any single object and plotting a long-term lightcurve fit to the instability model is not possible. For a typical supermassive black hole of $10^{8} M_{\odot}$, this would require timescales of hundreds of years in case of the radiation pressure and millions of years for the ionization instability driven evolution.

Nevertheless, statistical studies may shed some light on the sources evolution. For instance, the Giga-Hertz Peaked quasars, [3], have very compact sizes which would directly imply their ages on the order of 100-1000 years. However, there are too many of such sources to be all very young. Statistically, we would have too many young 
sources as compared to the mature quasars. An alternative explanation is therefore that in fact these sources are not young, but we observe now a subsequent step of the evolution, i.e. a reactivated source. The sample studied in [2] consists of 70 sources. The activity duty cycles are for most of them consistent with the sources ages, determined with a rough accuracy from either kinematics or synchrotron spectra.

Another possibility to study the duty cycles of quasars is studying their morphology and searching for various kinds of distortions or discontinuities in the radio structures. These structures may reflect the history of the central power source of a quasar, which went through the subsequent phases of activity and quiescence. An exemplary source of that kind, quasar $1641+320$, was studied in [7] and found to exhibit multiple radio structures. The source image is shown in Figure 1, where the radio structures labeled by $\mathrm{C}, \mathrm{E}, \mathrm{W} 1$ and $\mathrm{W} 2$ are the radio core, the eastern and the two western radio lobes, respectively. We suggest that the most recent jet direction is that from C to W2 structures in this source, while the other lobes originated from the past activity episodes.

An intermittent activity scenario can explain also the complex morphology of broad absorption line (BAL) quasar $1045+352$ [8]. The radio structure of $1045+352$ is dominated by the strong radio jet resolved into many sub-components and changing the orientation during propagation in the central regions of the host galaxy. As a consequence, we observe at least three phases of jet activity indicate different directions of the jet outflow. The source image is shown in Figure 3, where the radio structures labelled by $\mathrm{C}, \mathrm{A}, A_{1}, A_{2}$ and $\mathrm{B}$ are the radio core and the parts of the jet, respectively. We suggest that the current activity direction is the jet A emerging from the core to the east, while the other components probably originated from the past activity episodes.

Still, the best studied example of the radiation pressure instability in action is the microquasar GRS $1915+105$, which in some spectral states exhibits cyclic outbursts of X-ray luminosity, well fitted to a limit cycle oscillations of an accretion disk. This source is known for 20 years now, and only recently yet another microquasar of that type was discovered [1].

All above, and some other observational hints, discussed also in more detail in [5], support the existence of the thermal and viscous instabilities of accretion disks. Nevertheless, there exist many sources accreting at high accretion rate to the Eddington rate ratios, that do not exhibit the limit cycle oscillations on timescales adequate for the radiation pressure instability. Also, many AGN do not seem to exhibit cyclic activity. This may of course be a selection effect, or poor statistics in the observed lightcurves. On the other hand, some stabilizing mechanisms to the above discussed instabilities should be considered to explain the apparent stability of the high accretion rate sources.

\section{MECHANISMS OF THE DISK STABILIZATION}

The radiation pressure on the theoretical grounds has been verified by numerical tests and simulations (e.g., [12]).

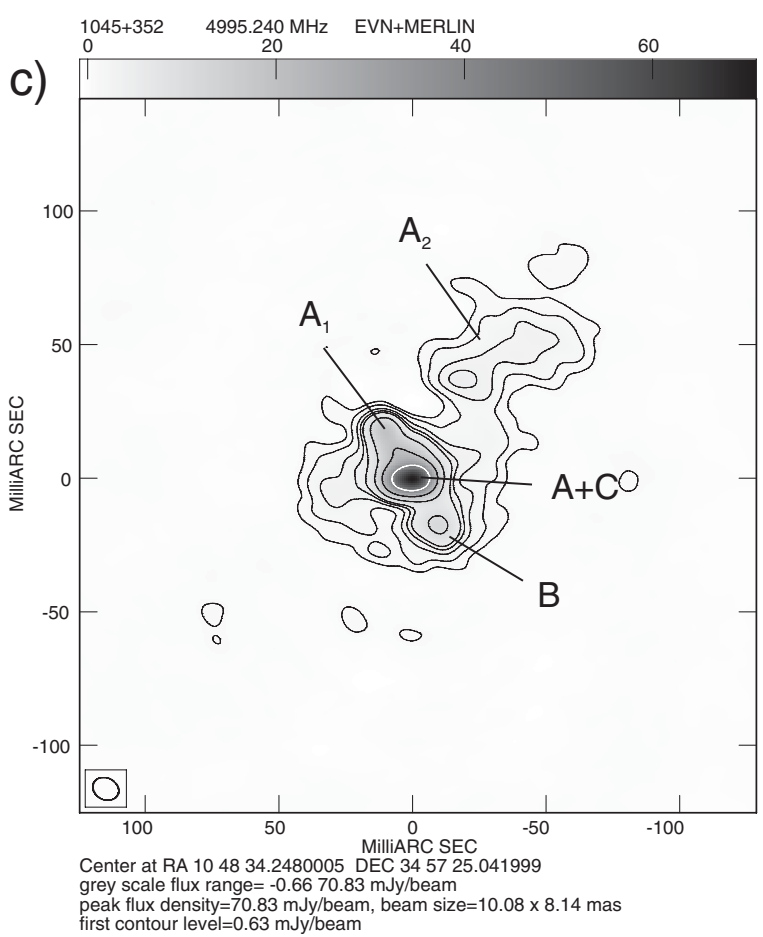

Figure 3. EVN radio image at $5 \mathrm{GHz}$ of the quasar $1045+352$ [8]. Contours increase by a factor of two and the first contour level corresponds to $\sim 3 \sigma$. The symbols indicate: $\mathrm{C}-$ radio core, $\mathrm{A}-\mathrm{A} 2$ - jet, B - counter-jet.

Two-dimensional simulations show that the radiation pressure in fact contributes to the total pressure in the viscous stress tensor, so such disk may be thermally and viscously unstable. On the other hand, the exact prescription for the disk heating law, either with a sum, or a geometrical mean of the gas and radiation pressures, is not certain. The former seems somewhat more natural and is elegant to use this simple form due to the Occam's razor. However, it has been shown that such a prescription leads in time dependent simulations to very strong periodic outbursts of the disk. Such outbursts by many orders of magnitude in luminosity are hardly observed, especially in AGN case.

One of the plausible mechanisms discussed in the literature that may affect the stability of accretion disks is the jet outflow. It has been shown that it correlates with the mass of an accreting black hole, giving an additional degree of freedom in the so-called 'fundamental plane' [11]. On this plane, the X-ray luminosity of a source correlates with its radio loudness which is the measure of the jet power. For AGN it may therefore result in stronger stabilizing effect on the radiation pressure dominated disks, even if the heating prescription is uniform for any mass of black hole. This supports the results of the simulations of accretion disk instabilities, where the jet power is incorporated in the model and provides additional source of cooling to the plasma [5].

Another effect that has been found recently [7] is a potential stabilizing effect of the companion star in a binary system or companion galaxy in case of quasars. The activity modulation due to the changes of the outer boundary conditions may be due to the periodically 


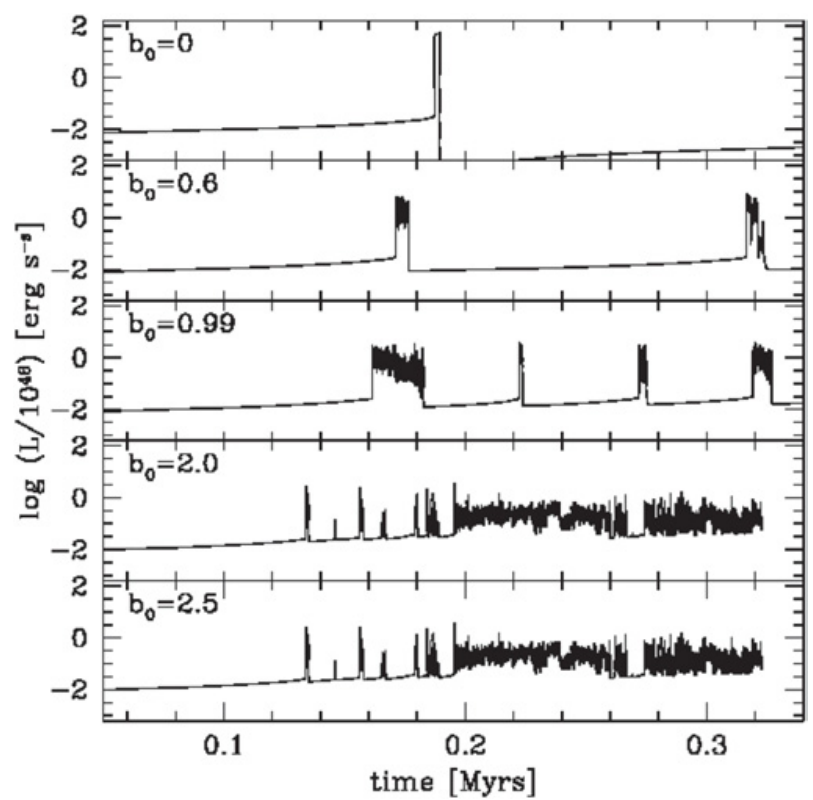

Figure 4. Theoretical lightcurves of the accretion disk in AGN under the limit cycle oscillations due to the radiation pressure instability (parameters of the model: black hole mass $M=3 \times 10^{8} M_{\odot}$, accretion rate $\dot{M}=0.33 \dot{M}_{\text {Edd }}$ and $\alpha_{0}=0.1$. The oscillations are gradually suppressed with the increase of the fluctuating part in the viscosity coefficient, as parametrized by the factor $b_{0}$.

changing external accretion rate, e.g. if the binary system orbit is eccentric. In this case the activity cycles are separated by prolonged low states, where the accretion rate in the disk drops below the level which is sufficient to trigger the instability. In addition, we found that if a variable energy transport to the jet is allowed, a prolonged high state appears between the limit cycle oscillations. This is an interesting discovery that could explain the effect of 'superoutbursts', observed in some sources.

Finally, what we found recently is that the viscosity fluctuations, modeled stochastically as a Markov chain process $[9,10]$ will lead to the ultimate viscous stability of radiation pressure dominated disk. This occurs when the fluctuating part in the viscosity coefficient is rather large.

In Figure 4 we plot the example of the limit cycle oscillations in the AGN accretion disk luminosity due to the radiation pressure. These outbursts are gradually stabilized by the fluctuating part in the viscosity coefficient, equal to $\alpha=\alpha_{0}\left(1+b_{0} u_{\mathrm{n}}(r, t)\right)$, where $u_{\mathrm{n}}$ is changing according to a Markov chain model, i.e. recalls the value of the $u_{n-1}$. The coherent length of these fluctuations is defined by the disk vertical thickness while their time is governed by the viscous timescale. During the activity cycles, a hot disk is geometrically thicker than a cold one, so the net effect requires numerical modeling and global, time-dependent simulations. Still, the thermal fluctuations in such models remain and the resulting lightcurves of accreting sources exhibit stochastic variability on very short timescales. The power density spectra approximately scale inversely with frequency and may be compared with observed variable sources, if the lightcurves are appropriately long and time-resolved.

\section{SUMMARY}

In accretion disks we can have two main types of thermalviscous instabilities:

- Radiation pressure instability

- Partial hydrogen ionization instability.

The radiation pressure instability can lead to the short term limit cycle oscillations in black hole x-ray binaries (tenshundreds seconds scales) or to cyclic activity of quasars (scales of tens-thousands of years). The ionization (or dwarf-nova type) instability can lead to the X-ray novae eruptions (scales of months-years) or long-term activity cycles in AGN (scales of millions of years).

The disk can be stabilized by:

- Very strong jet/outflow

- Heating prescription

- Companion

- Viscous fluctuations.

\section{References}

[1] Altamirano D., et al., ApJL 742, (2011) 17

[2] Czerny B., Siemiginowska A., Janiuk A., NikielWroczynski B., Stawarz L., ApJ 698, (2009) 840

[3] Fanti C., et al., A\&A 369, (2001) 380

[4] Janiuk A., Czerny B., Siemiginowska A., ApJL 542, (2000) 33

[5] Janiuk A., Czerny B., MNRAS 414, (2011) 2186

[6] Janiuk A., Misra R., A\&A 540, (2012) A114

[7] Kunert-Bajraszewska M., Janiuk A., ApJ 736, (2011) 125

[8] Kunert-Bajraszewska M., Janiuk A., Gawronski M.P., Siemiginowska A., 2010, ApJ, 718, 1345

[9] King A.R., Pringle J.E., West R.G., Livio M., MNRAS 438, (2004) 111

[10] Lyubarskii Y.E., MNRAS 292, (1997) 679

[11] Merloni A., Koerding E., Heinz S., Markoff S., DiMatteo T., Falcke H., New Astronomy 11, (2006) 567

[12] Ohsuga K., ApJ 640, (2006) 923 